\title{
INTEGRAL results on gamma-ray bursts and soft gamma-ray repeaters
}

\section{Sandro Mereghetti*}

INAF - IASF Milano, Italy

E-mail: sandrodiasf-milane.inaf.it

Despite not being specifically designed for the study of gamma-ray bursts (GRBs), the INTEGRAL satellite is giving a relevant contribution to this field with the detection of $\sim 10$ GRB year $^{-1}$ in the IBIS field of view. The ground-based INTEGRAL Burst Alert System (IBAS) has provided real-time localizations with 2-3 arcmin accuracy for most of these events. The INTEGRAL sample now comprises 81 bursts, including 4 of the short class. IBAS has also revealed other transient events from various classes of Galactic sources, most notably short bursts and flares from magnetar candidates. Here I present a summary of the IBAS performances in the eight years since the INTEGRAL launch and review some of the INTEGRAL results for GRBs and Soft Gamma-ray Repeaters.

8th INTEGRAL Workshop "The Restless Gamma-ray Universe”

September 27-30 2010

Dublin Castle, Dublin, Ireland

${ }^{*}$ Speaker. 


\section{Introduction}

INTEGRAL was successfully launched on 17 October 2002. On November 25, less than one month after the switch-on of its main instruments, INTEGRAL detected its first Gamma-ray Burst in the IBIS field of view (GRB 021125 [ए]]). A few weeks later the first GRB localization in real time was obtained for GRB 021219 [22]. These results were not unexpected, since pre-launch estimates [ㅁ] predicted a rate of $\sim 1 \mathrm{GRB} /$ month in the 0.23 sterad field of view of IBIS.

Up to now (January 2011), 81 GRBs occurred in the IBIS field of view ${ }^{1}$. Most of them were discovered in near real time by the INTEGRAL Burst Alert Systems (IBAS, [23]), resulting in the prompt distribution of their positions with accuracy of $\sim 2-3$ arcmin. IBAS has also revealed transient events from other classes of sources, like type I X-ray bursts from low mass X-ray binaries, short bursts and flares from magnetar candidates, and outbursts from other galactic transients. Here I briefly review some of the INTEGRAL results for GRBs and Soft Gamma-ray Repeaters localized with IBAS.

\section{Gamma-ray Bursts}

\subsection{IBAS performances}

The IBAS software for GRB detection runs in real time at the INTEGRAL Science Data Center (ISDC [ [6]), exploiting the fact that the satellite data are continuously downloaded and received at the ISDC without significant delays. IBAS started routine operations in April 2003, after a few months of testing and parameter tuning during which three GRBs were detected, but the automatic delivery of their positions was not yet enabled. On May 1st, the coordinates of GRB 030501 [D], with an uncertainty of only 4 arcmin were distributed $\sim 30 \mathrm{~s}$ after the start of the burst (see Fig. Ш). This was in absolute the first GRB with a small error region distributed in near real time (Swift became fully operational in the first months of 2005).

Rapid localizations have been obtained for $\sim 60$ INTEGRAL GRBs, while in other $\sim 20$ cases the coordinates have been distributed after some delay because, to avoid generating too many false alerts, the threshold for automatic distribution is kept at a conservative level. The triggers below such a threshold are checked with an interactive analysis, and indeed for most of them it is not possible to distinguish between a statistical fluctuation of the background and a genuine astrophysical event. However, several real GRBs were found in these off-line analysis and their coordinates distributed with typical delays of a few hours. Since February 2011 the sky coordinates also for low significance IBAS triggers are distributed in real time to interested users.

The Optical Monitoring Camera (OMC) on board INTEGRAL covers only the central $5^{\circ} \times 5^{\circ}$ around the satellite pointing direction, providing images of predefined CCD windows at the positions of known sources. When IBAS finds that a GRB is in the OMC field of view, a telecommand is automatically uploaded to the OMC in order to add a new CCD window covering the burst position. Up to now, this happened only for GRB 050626 , which unfortunately was at only $\sim 3$ arcmin from $\alpha$ Crucis and the OMC data were completely saturated by this very bright star.

\footnotetext{
${ }^{1}$ See online list at http://ibas.iasf-milano.inaf.it/IBAS_Results.html
} 


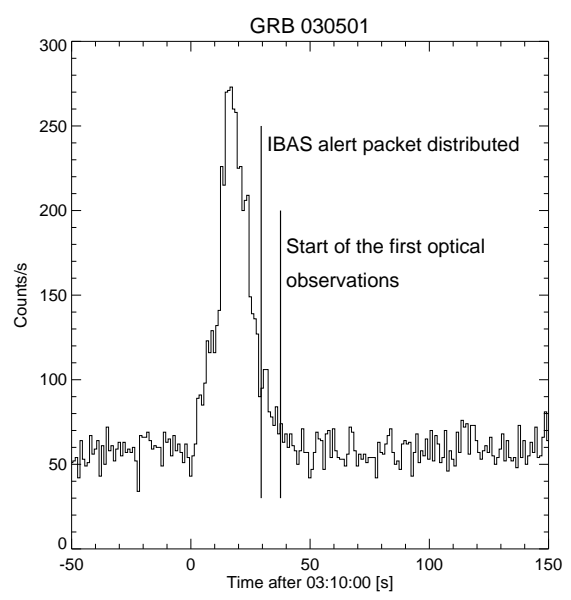

Figure 1: GRB 030501, the first GRB with an arcmin localization distributed in real time.

IBAS also reveals GRBs in the light curves produced by the Anti-Coincidence Shield (ACS) of the SPI instrument, which, besides serving to reduce the background in the germanium spectrometer, is routinely used as a nearly omni-directional detector for GRBs [4]]. It produces light curves with a resolution of $50 \mathrm{~ms}$ for photon energies $\gtrsim 80 \mathrm{keV}$, but without energy and directional information. These lightcurves are used for GRB triangulations with other satellites.

\begin{tabular}{|l|c|c|}
\hline GRB & redshift & reference \\
\hline 031203 & 0.1055 & {$[$ [B] $]$} \\
050223 & 0.584 & {$[$ [28] } \\
$050502 \mathrm{~A}$ & 3.793 & {$[\mathrm{BZ}]$} \\
$050525 \mathrm{~A}$ & 0.606 & {$[\mathrm{~B}]$} \\
080603 & 1.688 & {$[\mathrm{DQ}]$} \\
\hline
\end{tabular}

Table 1: INTEGRAL GRBs with spectroscopic redshift

\subsection{Global properties of the GRBs detected with IBIS}

The sky positions of the 81 GRBs located inside the IBIS field of view are shown in galactic coordinates in Fig.D. The anisotropy in their distribution is due to the non-uniform sky coverage of the INTEGRAL pointing program, mostly devoted to the Galactic bulge and plane. Only four of the INTEGRAL bursts belong to the class of short GRBs. This is a much smaller fraction than in the BATSE sample, where short GRBs accounted for about one fourth of the whole population, and is more similar to the findings of other instruments with a greater sensitivity at low energy than BATSE, like Swift/BAT (short GRBs account for $\sim 10 \%$ of the BAT sample).

Afterglows have been detected for more than half of the INTEGRAL GRBs: 41 at X-ray energy, 21 in the optical, and 3 in the radio band. Virtually all the GRBs for which follow-ups were carried out with XMM-Newton or Swift had an X-ray afterglow detected. Optical observations were in some cases discouraged by the low Galactic latitude. This possibly explains the relative paucity of optical afterglows in the INTEGRAL sample, but in some cases really deep upper limits were obtained (e.g. GRB 040223, GRB 040624 [प], and GRB 040403 [ㄷ]]). 


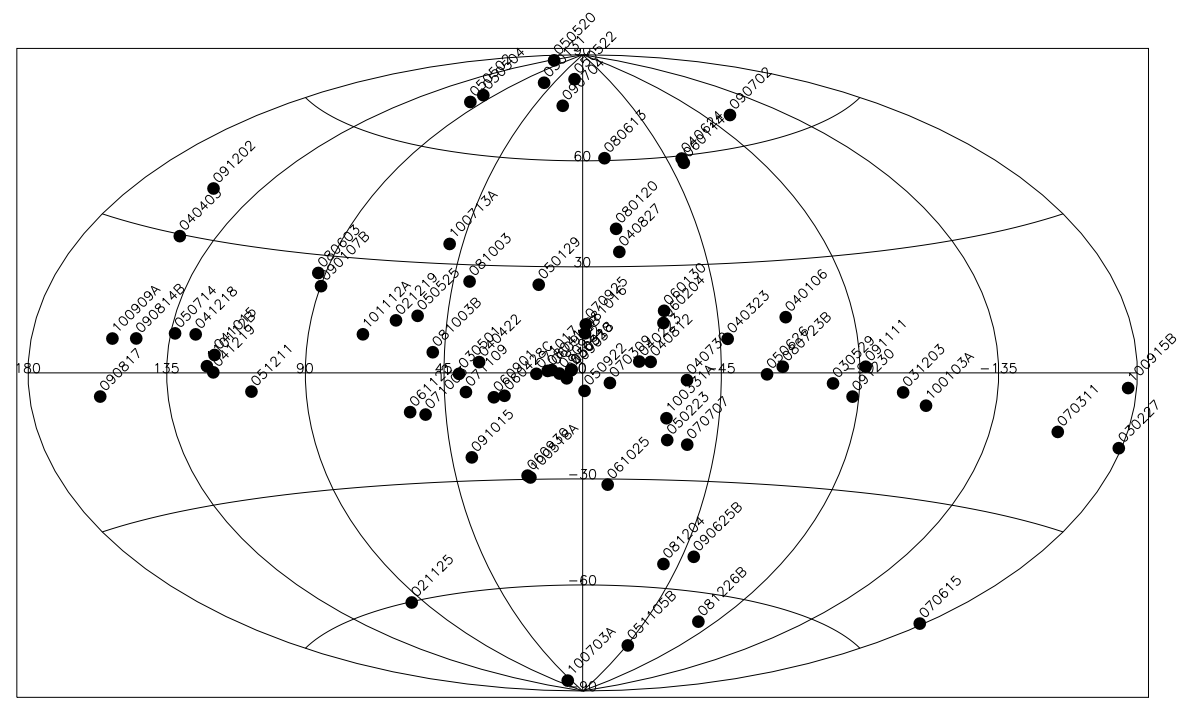

Figure 2: Positions in galactic coordinates of the GRBs detected with INTEGRAL/IBIS.

Spectroscopic redshifts have been obtained for 5 INTEGRAL GRBs (Table 1). GRB 050502 is the INTEGRAL GRB with the highest redshift. Its optical afterglow was detected only $\sim 23 \mathrm{sec}$ onds after the IBAS trigger and follow-up spectroscopy with the Keck telescope yielded a redshift of $z=3.793$ [32]. GRB 031203, at $z=0.1$, was one of the first GRBs with a spectroscopic supernova association, and is discussed in more detail below.

A systematic analysis of all the bursts detected with IBIS before mid 2007 [40] shows that, from the spectral point of view, they do not differ significantly from the larger sample of Swift/BAT GRBs. In the relatively narrow energy band covered by the ISGRI/IBIS detector, their spectra are generally well fit by a power law with average photon index $\sim 1.6$. This value is intermediate between the average low-energy and high-energy slopes obtained with the broken power law model usually adopted for GRBs, suggesting a spectral break within the IBIS/ISGRI range. Indeed this is confirmed for the few bright bursts with sufficient statistics, for which an exponentially cut-off power-law gives a significantly better fit and the average cut-off energy is $\sim 80 \mathrm{keV}$. On the other hand, in a few cases the steep power-law spectra measured by ISGRI (photon index $>2.5$ ) indicate that the event can be classified as an X-ray Flash (e.g. GRB 030529 and 040903 [40]).

The peak fluxes of the GRB revealed by IBIS range from $\sim 0.08 \mathrm{ph} \mathrm{cm}^{-2} \mathrm{~s}^{-1}$ to $>50 \mathrm{ph}$ $\mathrm{cm}^{-2} \mathrm{~s}^{-1}$ (20-200 keV, $1 \mathrm{~s}$ integration time). The upper limit is uncertain because bright fluxes saturate the telemetry allocated to IBIS. A possible difference between the INTEGRAL and Swift GRB samples is suggested by the comparison of their brightness. A probability of only $3 \%$ that the IBIS and BAT peak fluxes are drawn from the same distribution was found [40], indicating that IBIS, on average, reveals fainter GRBs than BAT. This is consistent with the smaller field of view of IBIS, giving a higher sensitivity near the on-axis direction, at the expenses of a lower rate of detections.

The spectral lag for a sample of INTEGRAL GRBs was computed by [Q], who found that the subclass of faint bursts with long spectral lag are spatially correlated with the Supergalactic plane. This has been interpreted as evidence for a local population of low luminosity GRBs, a result 
questioned by [42] on the basis of the Swift sample. Although a reanalysis of the INTEGRAL data, accounting for the IBAS selection effects that were neglected in [Q], supports the presence of this spatial correlation [40], a larger sample is needed to unambiguously confirm the existence of such a local population.

\subsection{GRB 031203}

GRB 031203 is among the most interesting GRBs discovered by INTEGRAL. Its afterglow was observed at X-ray, IR/Optical, and radio wavelengths, and spectroscopic evidence of an associated Type Ic Supernova was found [एष]]. Its 20-200 keV fluence of $\sim 10^{-6} \mathrm{erg} \mathrm{cm}^{-2}$ and low redshift $(\mathrm{z}=0.1)$ imply an isotropic-equivalent energy $\mathrm{E}_{\text {iso }}$ of only a few $10^{49} \mathrm{erg}$, three orders of magnitude below that of normal GRBs [[33], and more typical of an X-ray flash. On the other hand, as confirmed in our re-analysis of the IBIS data with recent software and calibrations [40], GRB 0312013 had a hard spectrum well fit by a power-law with photon index $\sim 1.5$, and a 99\% c.l. lower limit of $100 \mathrm{keV}$ was derived on its $\mathrm{E}_{\text {peak }}$. These results make GRB 031203 an outlier of the correlations followed by most GRBs [ [ [1]].

The X-ray images obtained with XMM-Newton a few hours after the burst led to the discovery of expanding rings due to the scattering of the GRB X-ray emission by dust grains in our Galaxy [B8]. This "echo", the first one observed around a GRB, provides an indirect way to estimate the intensity of the prompt $\mathrm{X}$-ray emission, which is found to exceed the extrapolation to a few $\mathrm{keV}$ of the INTEGRAL spectrum [38], 36]. Thus it is likely that the soft X-ray emission in GRB 031203 consisted of a delayed component following the hard pulse seen with INTEGRAL, similar to what has been observed in GRB 060218 [ए2]. In this case GRB 031203 would have a smaller $\mathrm{E}_{\text {peak }}$ and a higher luminosity, making it in agreement with the "standard" relations.

\subsection{GRB 041219A}

GRB 041219A, the longest and one of the brightest GRB localized by IBAS, was composed of one weak precursor followed, after a quiescent interval lasting $\sim 4$ minutes, by the main GRB emission consisting of two bright pulses. Thanks to the IBAS trigger and localization on the faint precursor, successful optical and near IR observations started while the burst was still on going [B], [39]. The prompt optical and gamma-ray emission were correlated, suggesting that both components originated in the internal shock, contrary to the only previously observed case, GRB 990123, in which the optical prompt emission was anticorrelated with the high-energy light curve, and was interpreted as arising in the reverse shock.

The large fluence of GRB 041219A prompted searches for polarization exploiting Compton interactions in the INTEGRAL instruments. Hints for the presence of linear polarization above

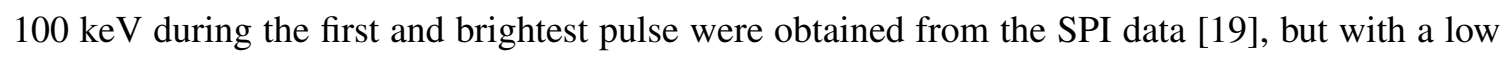
statistical significance. These findings were only partially confirmed with IBIS [ㄷ]], which, on the other hand, showed a possible linear polarization of the second pulse. The analysis of IBIS data seems also to indicate that the polarization varies, both in intensity and angle, during the burst. Although these results are tantalizing and have potentially very interesting implications for GRB theories, they are based on complicated and non-standard analysis, and their real significance, which also should properly take into account the number of trials, is not easy to assess. 


\subsection{GRB 070707}

GRB 070707 was the first short GRB detected by INTEGRAL. It had a duration of $0.8 \mathrm{~s}$, a spectrum well described by a power law with photon index 1.2, and a small spectral lag of $20 \pm 5$ ms [16]. These characteristics are typical of the class of short-hard bursts. An X-ray afterglow was discovered with Swift, permitting the identification of a faint $(\mathrm{R} \sim 23)$ optical transient. The optical flux initially decayed very steeply, and then leveled at $\mathrm{R} \sim 27$ after a few days. If, as it is likely, the constant flux is due to the host galaxy, this is one of the faintest revealed for a short GRB [BO]].

\section{Soft Gamma-ray Repeaters}

Soft gamma-ray repeaters (SGRs) are X-ray sources characterized by the emission, during sporadic periods of activity, of short $(<1 \mathrm{~s})$ bursts of soft gamma-rays with peak luminosity up to $10^{42} \mathrm{erg} \mathrm{s}^{-1}$, and, much more rarely, by the occurrence of giant flares, releasing up to $10^{46} \mathrm{ergs}$. SGRs are well explained as magnetars: isolated neutron stars powered by extremely high magnetic fields, $B>10^{14}-10^{15} \mathrm{G}$ [B5]]. Short bursts have been detected also from most Anomalous X-ray Pulsars (AXPs, [R]]), thus supporting their interpretation as another class of magnetars. The most recent observations of AXPs and SGRs suggest that their different classification merely reflects the way they were originally discovered (see [26] for a review).

\subsection{SGR 1806-20}

SGR $1806-20$ is located at only $10^{\circ}$ from the Galactic Center direction, a sky region extensively observed by INTEGRAL, and has been one of the most active SGRs after the INTEGRAL launch. More than 300 bursts have been detected, of which about 100 occurred within 10 minutes of exceptionally high activity on October 5, 2004 [ㅍ]]. The high sensitivity of IBIS allowed us to detect the faintest bursts ever observed from this SGR in the 20-100 keV energy range, reaching fluences below $\mathrm{S} \sim 10^{-8} \mathrm{erg} \mathrm{cm}^{-2}$. The $\log \mathrm{N}-\log \mathrm{S}$ of the bursts is consistent with a single power law with slope $0.91 \pm 0.09$, independent of the bursting rate. Time resolved spectroscopy of some of the brightest bursts showed significant spectral evolution, generally with the softest emission at the peak [피].

The giant flare emitted from SGR 1806-20 on 2004 December 27 was the brightest event of this class ever observed, and it was first reported thanks to its detection with IBAS in the SPI/ACS data [ [9]. While all the other satellites could observe the giant flare only for a few minutes before it faded below their sensitivity, the ACS detected high-energy emission ( $>80 \mathrm{keV}$ ) from 1E 1547.05408 lasting about one hour after the giant flare [24]. This component, observed for the first time in a magnetar giant flare, has been interpreted as a hard X-ray afterglow produced by the matter ejected relativistically, with bulk Lorentz factor $\Gamma \sim 15$ during the initial spike at the beginning of the flare.

\subsection{E 1547-5408}

Although known since more than 30 years, the transient X-ray source 1E 1547.0-5408 was recognized as a member of the magnetar class only recently, after the discovery of radio and X-ray pulsations with a period of $2.1 \mathrm{~s}$ and period derivative $\dot{P}=2.3 \times 10^{-11} \mathrm{~s} \mathrm{~s}^{-1}$, and the observation of SGR like bursts [10, 回]. 
In January 2009 1E 1547.0-5408 entered a new phase of bursting activity, during which many short bursts of soft gamma-rays were emitted. The period with the highest bursting rate was observed with the ACS, which provided a continuous, uninterrupted coverage from 14:35 UT of January 20 to 04:23 UT of January 23. Two of the bursts showed tails lasting several seconds and modulated at the spin period of 1E 1547.0-5408 resembling the pulsating tails seen after giant flares [27, [34]. However, the energetics of these two bursts $\left(\sim 10^{43} \mathrm{erg}\right.$ for $\left.\mathrm{d}=5 \mathrm{kpc}\right)$ was not as large as that of giant flares.

The peak of the bursting rate occurred around 6:48 UT of January 22, when more than 50 bursts were recorded in 10 minutes. The soft X-rays emitted during this short time interval were scattered by three clouds of interstellar dust, producing expanding halos around the source that were imaged with Swift/XRT and still detectable with XMM-Newton after twelve days [B7]. Since the time evolution of the halo flux and angular size depend on the distances of the source and of the scattering clouds, it was possible to derive the distance of 1E 1547.0-5408 and to get some constraints on the dust properties and distribution from an analysis of the X-ray data. Although the source distance depends on the adopted dust model, the best fits favored values of $\sim 4-5 \mathrm{kpc}$, supporting the association of 1E 1547.0-5408 with the supernova remnant G 327.24-0.13.

\section{Conclusions}

Although INTEGRAL was designed as a multi-purpose gamma-ray Observatory, not specifically optimized for GRBs, it has significantly contributed to this field thanks to its excellent imaging performances and good sensitivity. INTEGRAL unique features include the capability of long uninterrupted monitoring with the SPI/ACS and the possibility to carry out polarization searches exploiting Compton interactions in the multiple detectors. Unfortunately, some of the potentials for INTEGRAL GRBs studies are hampered by limitations in the satellite telemetry, which reduce the available count statistics for the brightest events. The recent implementation of IBAS alert distribution for low significance triggers should lead to a higher rate of INTEGRAL GRBs, possibly confirming the presence of a local population of GRBs and/or leading to the discovery of more bursts at high redshift.

Acknowledgments. I acknowledge all the scientists and engineers who participated to the IBAS design, in particular Jurek Borkowski and Diego Götz for their invaluable contribution to its realization and operation. Smooth running of IBAS has been possible thanks to M. Beck, V. Beckmann, N. Mowlavi, C. Ferrigno, E. Bozzo and all the operational staff of the ISDC.

\section{References}

[1] Amati, L., Frontera, F., Tavani, M., et al. 2002, A\&A, 390, 81

[2] Beckmann, V., Borkowski, J., Courvoisier, T. J.-L., et al. 2003, A\&A, 411, L327

[3] Blake, C. H., Bloom, J. S., Starr, D. L., et al. 2005, Nature, 435, 181

[4] Borkowski, J., Gotz, D., Mereghetti, S., Mowlavi, N., Shaw, S.,\& Turler, M. 2004, GRB Coordinates Network, 2920, 1

[5] Camilo, F., Ransom, S. M., Halpern, J. P., \& Reynolds, J., ApJ, 666, L93, 2007 
[6] Courvoisier, T. J.-L., et al. 2003, A\&A, 411, L53

[7] Filliatre, P., et al. 2006, A\&A, 448, 971

[8] Foley, R. J., Chen, H.-W., Bloom, J., \& Prochaska, J. X. 2005, GRB Coordinates Network, 3483, 1

[9] Foley, S., McGlynn, S., Hanlon, L., McBreen, S., \& McBreen, B. 2008, A\&A, 484, 143

[10] Gelfand, J. D. \& Gaensler, B. M., ApJ, 667, 1111, 2007

[11] Ghirlanda, G., Ghisellini, G., Lazzati, D., \& Firmani, C. 2004, ApJ, 613, L13

[12] Ghisellini, G., Ghirlanda, G., Mereghetti, S., et al. 2006, MNRAS, 372, 1699

[13] Götz, D., Mereghetti, S., Mirabel, I. F., \& Hurley, K. 2004, A\&A, 417, L45

[14] Götz, D., Mereghetti, S., Molkov, S., et al., A\&A, 445, 313, 2006

[15] Götz, D., Laurent, P., Lebrun, F., Daigne, F., \& Bošnjak, Ž. 2009, ApJ, 695, L208

[16] McGlynn, S., Foley, S., McBreen, S., et al. 2008, A\&A, 486, 405

[17] Malaguti, G., Bazzano, A., Beckmann, V., et al. 2003, A\&A, 411, L307

[18] Malesani, D., Tagliaferri, G., Chincarini, G., et al. 2004, ApJ, 609, L5

[19] McGlynn, S., Clark, D. J., Dean, A. J., et al. 2007, A\&A, 466, 895

[20] Mereghetti, S., Cremonesi, D. I., \& Borkowski, J. 2001, ESA SP-459, 513

[21] Mereghetti, S., \& Stella, L. 1995, ApJ, 442, L17

[22] Mereghetti, S., Götz, D., Beckmann, V., et al. 2003, A\&A, 411, L311

[23] Mereghetti, S., Götz, D., Borkowski, J., Walter, R., \& Pedersen, H. 2003, A\&A, 411, L291

[24] Mereghetti, S., Götz, D., von Kienlin, A., et al. 2005, ApJ, 624, L105

[25] Mereghetti, S., et al. 2005, A\&A, 433, 113

[26] Mereghetti, S., A\&AR, 15, 225, 2008

[27] Mereghetti, S. et al., ApJ, 696, L74, 2009

[28] Pellizza, L. J., et al. 2006, A\&A, 459, L5

[29] Perley, D. A., Bloom, J. S., \& Prochaska, J. X. 2008, GRB Coordinates Network, 7791, 1

[30] Piranomonte, S., et al. 2008, A\&A, 492, 775

[31] Prochaska, J. X., Bloom, J. S., Chen, H.-W., et al. 2004, ApJ, 611, 200

[32] Prochaska, J. X., et al. 2005, GRB Coordinates Network, 3332, 1

[33] Sazonov, S. Y., Lutovinov, A. A., \& Sunyaev, R. A. 2004, Nature, 430, 646

[34] Savchenko, V., Neronov, A., Beckmann, V., Produit, N., \& Walter, R. 2010, A\&A, 510, A77

[35] Thompson, C. \& Duncan, R. C., MNRAS, 275, 255, 1995

[36] Tiengo, A. \& Mereghetti, S., A\&A, 449, 203, 2006

[37] Tiengo, A., et al., ApJ, 710, 227, 2010

[38] Vaughan, S., Willingale, R., O’Brien, P.T., et al., ApJ, 603, L5, 2004

[39] Vestrand, W. T., Wozniak, P. R., Wren, J. A., et al. 2005, Nature, 435, 178

[40] Vianello, G., Götz, D., \& Mereghetti, S. 2009, A\&A, 495, 1005

[41] von Kienlin, A., Beckmann, V., Rau, A., et al., A\&A, 411, L299, 2003

[42] Xiao, L., \& Schaefer, B. E. 2009, ApJ, 698, 803 